\title{
MÉDIAS ET INTERNET : RENOUVELLEMENT DE LA RELATION AVEC LES USAGERS OU ÉVOLUTION EN TROMPE-L' 'EIL ?
}

\author{
Serge Guérin ${ }^{1}$
}

Dans quelle mesure l'appropriation du réseau Internet par les médias traditionnels ${ }^{2}$ a-t-il transformé la relation entre l'organe de presse et son public ? A l'origine, les pionniers de la presse en ligne -comme David Talbot ancien du San Francisco Examiner qui fonde en 1995 le site Salon- entendaient aussi que cette nouvelle forme de diffusion de l'information contribue à créer un renouveau dans la relation journaliste/usagers ${ }^{3}$. Les médias sont d'abord des institutions sociales et permettent l'expression d'une pensée. Les médias traditionnels sont les premiers supports, type presse écrite et audiovisuel. Mais c'est l'usage qui transforme une technique en média. L'apparition d'Internet avec son potentiel d'interactivité relance et modernise le discours traditionnel du monde de la communication et

1 Journaliste, directeur de la rédaction du groupe Golding (France Graphique, docutique...). Professeur associé à l'Université de Lyon 2.

${ }^{2}$ F. Balle, Médias et Sociétés, Paris, Montchrétien, 9e édit., 2000. Pour Francis Balle, les médias sont d'abord des institutions sociales et permettent l'expression d'une pensée. Les médias traditionnels sont les premiers supports, type presse écrite et audiovisuel. Mais c'est l'usage qui transforme une technique en média.

3 S. GuÉRIN, La Cyberpresse, Paris, Hermes, 1995, p. 43.

Recherches en communication, $\mathrm{n}^{\circ} 21$ (2004). 
des "machines à communiquer" qui s'auto-légitime comme une source dialectique permettant un échange et un dialogue constant et multiple au travers des différentes strates du corps social ${ }^{1}$.

Si les modes de développement de l'information en ligne sont polymorphes et particulièrement évolutifs, on cherchera cependant, au-delà d'une tentative illusoire de description des expériences, à interroger les situations présentes pour déterminer en quoi ce nouveau support transforme les conditions du feed-back entre émetteurs et récepteurs.

\section{De la démocratisation technologique}

S'interroger sur les conséquences du développement de la communication sur le réseau pour la relation média et usagers revient pour une large part à s'inscrire dans la problématique issue des années 1980 où de nombreuses recherches se sont portées sur la dimension interactive des nouvelles technologies de communication. Des chercheurs comme Serge Proulx et Michel Sénécal nous ont invités à tenir pour différente l'interactivité technologique liée aux nouvelles possibilités de dialogue entre l'homme et la machine, et l'interaction sociale comprise comme l'accès de tous à un nouvel état de la communication. Selon ce courant ${ }^{2}$, la confusion entretenue entre ces deux lectures rend compte du faible gain de démocratisation qualitative de la communication générée par des nouvelles technologies.

Les NTIC ont aussi suscité un regard d'ordre anthropologique pour une analyse des relations qu'elles induisent. La notion de messageries, en particulier, a-t-elle produit de nouvelles formes de relätions ? De fait la nouvelle puissance de la communication à distance, le nouveau rapport au lien et son vécu d'ordre fantasmatique ont ouvert de nouvelles pratiques. Reste à savoir si ces relations virtuelles, d'autres diront fictives, participent de nouvelles formes de sociabilité et si elles les renforcent. C'est par exemple le point de vue de $M$. Maffesoli. D'autre comme A. Akoun estime que le succès des formes de communication électronique relève d'abord d'un comportement narcissique où l'émetteur et le récepteur tendent avant tout à se

1 Ph. Breton, L'utopie de la communication, Paris, La Découverte, 1995.

2 S. ProulX et M. SÉnÉCAL, L'interactivité technique, simulacre d'interaction sociale et de démocratie?, TIS, vol. $7 \mathrm{n}^{\circ}$ 2, 1995. Mais aussi Ph. BRETON, op cit. 
représenter l'autre (l'interlocuteur) à son image. L'éloignement permettrait de réduire le risque de se confronter au réel et permettrait une relation "cosmétique " qui a pour principale différence mais de faire l'économie des rugosités humaines.

On trouvera aussi des oppositions de perspectives concernant les usages sociaux de l'interactivité. Une approche, que l'on peut s'autoriser à qualifier d'optimiste, nous vient de Pierre Lévy ${ }^{1}$ sur "l'intelligence collective" autorisée par les nouvelles technologies. Il est ici possible de mettre en synergie la compétence des individus à travers leur mise en contact et en dialogue constructifs via le réseau des réseaux. Cette vision totémique du réseau laisse cependant la place à l'indéterminisme au sens où Pierre Lévy par exemple entend bien qu'elle dépende de l'usage que les individus et les sociétés font et feront de ces technologies. Mais le primat revient aux facteurs humains et sociaux et minore le poids des logiques économiques, des contraintes de profitabilité et des réalités du capital culturel dans l'intensité des échanges sociaux.

A cette modification de "la pragmatique de la communication" répond le souci de l'acteur ${ }^{2}$. L'isolement social ne semble pas régresser avec le développement des technologies de l'interactivité, en témoigne le développement des ménages d'une seule personne ou encore le maintien de la violence sociale et guerrière. De cette optique, l'utopie du village mondial laisse la place pour le moins à la ville mondiale, pour reprendre la distinction, qui dépasse la seule perspective sémiologique, produite par Z. Breszinsky.

Ainsi avec Victor Scardigli ${ }^{3}$, il apparait qu'il ne faut pas se laisser prendre par une certaine mystification selon laquelle les innovations des technologies de la communication portent en elles-mêmes un atout communicationnel et partant un nouveau lien entre les médias et les récepteurs.

Mais depuis que se développent les sites d'information et la mise en ligne de médias écrits ou audiovisuels, en quoi les relations avec les différents usagers ont-elles évolué ?

1 P. LÉVY, Les technologies de l'intelligence, Paris, Éd. du Seuil, 1993.

2 M. Crozier et E. FriedBerg, L'acteur et le système, Paris, Éd. du Seuil, 1977.

3 V.Scardigli, Les sens de la technique, Paris, PUF, coll. "Sociologie d'aujourd'hui", 1992. 


\section{Vers de nouvelles transversalités?}

Avant tout, il importe de rechercher à fournir une typologie même sommaire des différentes formes de mise en ligne des médias. Ce que nous avons appelé par ailleurs, la cyberpresse. Après différents essais et tentatives, il est possible de proposer quatre approches principales : le site vitrine, la duplication de tout ou partie du support d'origine, la complémentarité par rapport aux éditions traditionnelles associée à la multiplication d'interfaces et, enfin, l'orientation portail.

Dans les deux premiers cas, le développement d'interfaces favorisant le feed-back des usagers vers ceux qui se trouvent dépositaires du message est, lorsqu'il fonctionne et existe, réduit au minimum. Il s'agit presque exclusivement de la possibilité ouverte de s'abonner en ligne pour des produits de presse et pour des chaînes payantes, de pouvoir recevoir le sommaire de la nouvelle édition par e-mail, et de disposer de l'adresse électronique de la rédaction en général ou de chaque rédacteur en particulier.

Les sites qui entendent développer une offre complémentaire au média d'origine (prolongement des articles, des interviews ou des reportages par la mise à disposition de sources documentaires, d'images fixes et animées, d'extraits d'actualité, de liens avec d'autres sites plus exhaustifs sur la question...) proposent aussi des interfaces plus riches comme l'organisation de forum de discussion, le plus souvent avec modérateurs, à partir des sujets traités, des chat avec des responsables de la rédaction ou des personnalités invitées.

Les sites de médias qui multiplient les contenus plus larges, à l'image de webdo issu du news suisse L'Hebdo qui proposait pratiquement dès sa première version, d'autres supports mais aussi des lettres spécifiques et des contributions d'auteurs distinctes de la production d'origine, développent les mêmes formes d'interface que précédemment.

En revanche, ils y ajoutent des espaces d'interventions pour les usagers à travers principalement des forums de discussion liés à des thèmes traités dans les éditions "réelles", des FAQ (foires aux questions) ou la possibilité d'adresser son opinion. 


\section{Vers le renouveau de la relation usagers-médias ?}

La sensibilité aux réactions des lecteurs est une constante de la presse moderne ${ }^{1}$. En soi, le système des médias s'inscrivant dans une logique ultra-concurrentielle ${ }^{2}$, l'attention à la réaction du public est une obligation, une condition de survie.

L'achat du titre de presse ou les études d'audience en radio et en télévision restent le premier baromètre des réactions des usagers des médias et fonctionnent comme un outil de mesure de la satisfaction des lecteurs, auditeurs ou téléspectateurs ${ }^{3}$, mais le courrier des lecteurs tient un rôle à la fois plus impliquant pour le journaliste comme pour celui qui en est l'auteur. Dans les salles de rédaction, il est traditionnellement d'usage d'estimer qu'un mouvement hors norme du nombre de lettres reçues est un signe fort, quel que soit le nombre total de ces lettres, d'une réaction, positive comme négative, du public. Ici le nombre ne fait pas sens en lui-même, mais est porteur d'un fait objectivé. Pour approuver, ou au contraire réprouver, un article, une prise de position spécifique du média, ou encore l'occultation d'un fait, chacun peut entreprendre de s'adresser à un journaliste ou à un responsable de rédaction (gate-keeper) ${ }^{4}$, pour exprimer une réaction. Celui qui "ose" prendre sa plume, qui fait l'effort de rédiger un courrier et de l'adresser est porteur d'une sur-motivation qui lui permet de dépasser les contraintes du quotidien et de ses propres inhibitions (ne serait-ce que par la peur culturelle du jugement de l'écrit). Il est donc représentatif d'un sentiment partagé par d'autres et surtout d'un sur-sentiment.

Or, la possibilité offerte à l'usager du média de réagir via e-mail supprime une série de barrières comme le rapport à l'écrit ou la nécessité d'accomplir des formalités pour l'envoi postal et s'inscrit dans l'ordre de l'instantané. Le plus souvent la lettre impose un recul et l'acceptation d'une norme sociale intégrant par exemple des formules de politesse et la recherche d'une construction de raisonnement, à l'inverse le mail permet de s'affranchir pour une grande part

1 J.-M. Charon, La presse en France, Paris, Éd. du Seuil, 1991.

2 F. BALLE, op cit.

3 M. MAthiEn, Le système médiatique. Le journal dans son environnement, Paris, Hachette, 1989, pp. 93-96.

4 M. Mathien, op cit. 
de ces règles pour privilégier une forme d'oralisation qui tend à donner l'impression que les barrières culturelles et hiérarchiques sont abaissées. Une simple question peut ainsi être posée sans artifices supplémentaires, de même qu'une réaction peut s'exprimer "brute de fonderie" et dans l'urgence si nécessaire.

Le feed-back est donc en théorie facilité avec la possibilité offerte d'échanger par les voies électroniques. En fait, il apparaît bien que cela ne transforme pas profondément la nature de la relation entre usagers et média car même si la création de sites ou d'adresses e-mail a globalement favorisé la réactivité des usagers, les auteurs restent dans le même registre, en termes de contenu comme d'origine, que ceux passant par le prisme du courrier "traditionnel".

De la même façon, les journalistes à qui s'adressent les e-mail n'ont pas de façon sensible changé leur mode de réponse. Une fois passé la première curiosité et le phénomène de mode, ce qui suscite la réponse tient aux. mêmes réalités que pour le courrier : intérêt du message, nature de la demande, temps disponible. Le fait de pouvoir répondre dans l'instantanéité facilite certes fortement le retour mais ce nouveau confort est contrecarré par la profusion de mail de diverses origines. Tous les rédacteurs se trouvent en effet confrontés à la même problématique : le manque de temps pour lire et répondre à une inflation de messages reçus sur leurs écrans. Ce n'est pas tant le nombre de courriers électroniques adressés par des usagers, très inférieur aux premières attentes, que la concurrence avec d'autres messages électroniques reçus qui contribuent à ce que moins de $10 \%$ des envois d'usagers génèrent une réponse personnalisée.

On notera en outre que le recours à la messagerie électronique n'est pas en soi un gage de pertinence dans l'énoncé de la question ou de la réaction. Au contraire. Il apparaît que la facilité d'utilisation permet le développement d'un flux, d'une prise de parole qui se satisfait le plus souvent du caractère informel de la relation, on peut estimer qu'il s'agit d'une oralisation du message, pour ne pas chercher à structurer l'intervention. Comme si le médium utilisé n'avait pas suffisamment de sacralité pour forcer celui qui entreprend de prendre la parole et d'interpeller le média à défendre son point du vue aux moyens d'arguments cohérents et réfléchis et d'un mode d'expression qui réponde à une norme sociale définie.

De ce point de vue, le développement des messageries électroniques comme mode de relation entre un média et ses usagers entraîne, dans une mesure très relative, un surcroît quantitatif dans le 
feed-back créé, mais qui ne se retrouve qu'imparfaitement dans le "qualitatif" des messages.

Autrement dit, il importe de porter rapidement l'analyse sur le contenu des messages. Il n'y a pas eu d'âge d'or', du courrier des lecteurs, électronique ou non ; ni en termes de quantité, le plus souvent fonction de l'actualité ou du décalage entre l'énoncé du discours et la vision de la réalité traditionnellement émise par le public récepteur, ni en termes de qualité, pour autant qu'il en soit possible de proposer une vision normative. Mais l'ensemble des entretiens menés avec des journalistes montre que l'accès au numérique a fait évoluer le type d'intervention des usagers des médias dans la sphère médiatique. Les interventions des usagers via Internet se distinguent du courrier traditionnel par une proportion beaucoup plus importante de questionnement, de demande de précision. On assiste presque en direct à la formulation par le lecteur ou l'auditeur de ses interrogations. Ici la relation au médiateur est désacralisée, celui-ci devient une sorte de conseiller dont la légitimité ne se fonde pas sur le statut, la carte de presse et la signature dans un support donné, mais sur la capacité à tenir un rôle de veilleur, à assurer la description et la mise en perspective d'une série de faits ou de permettre la transmission d'un savoir. Cette relation n'est pas une forme de dialogue égalitaire mais le moyen d'aller un peu plus au fond des choses par le questionnement.

L'autre vecteur d'intervention de l'usager dans le quotidien du journaliste c'est l'apostrophe, la réaction vive et l'envie de marquer son assentiment ou son désaccord. La facilité d'utilisation du courrier électronique et sa rapidité de mise en cuvre favorise et libère l'expression d'opinions et de sentiments. Il permet aussi l'invective. Le médium est structurant : le courrier électronique par sa simplicité d'usage et sa réactivité se positionne plus dans l'ordre de la conversation que dans le domaine de l'échange épistolaire. C'est donc un facteur de désinhibition.

Est-ce pour autant, là aussi du dialogue ? Pas réellement car l'usager n'attend pas une réponse, ni un échange mais se situe plutôt dans une posture de dénonciation ou d'appréciation. Il porte un jugement plus qu'il ne se place dans l'ordre de la raison et de la confrontation d'idées et d'arguments.

1 J.-M. Charon, op cit. 
Le courrier électronique favorise bien de nouveaux feed-back, une nouvelle façon de réagir et de "consommer" du média, sans pour autant que cela traduise nécessairement un rapprochement des points de vue des usagers et des médias.

La multiplication de ces feed-back permet cependant de poser l'hypothèse de la contribution de la mise en ligne de médias au rapprochement avec le collectif des récepteurs. La mise en ligne agit comme un vecteur de rapprochement, d'intégration plus profonde, dans le quotidien, et dans la sphère sociale. Cela est d'autant plus facilité que l'usage de l'information en ligne se pratique au sein même du temps alloué au travail. A l'inverse de la consommation des médias "réels" qui sauf exception ne trouve pas place dans la journée de travail, mais plutôt l'encadre à travers les temps de transports et les activités de loisirs, le site de presse peut facilement être consulté sur le lieu de travail. Une partie non négligeable des usagers pratiquant ces consultations uniquement par ce biais.

Restent que ces pratiques, qui sont cependant seulement pionnières, ouvrent un nouveau champ à l'intervention de l'usager dans le cour du dispositif médiatique. Elles contribuent à y faire rentrer de nouvelles interrogations, à forcer le média à prendre en compte la réalité du ressenti de son public. Sa (re)construction du réel en est ainsi transformée.

\section{Interactivités : nouvelles formes d'implication de l'usager}

Mais les feed-back proposés par les sites de médias ouvrent aux usagers d'autres possibilités d'expression. La plus importante tient au développement des forums de discussion proposés par les sites ou abrités par eux. Ces forums recréent une agora, une place de village, légitimée par la marque de presse. Selon les cas, ces forums naissent d'un sujet abordé par le média ou se forment par une communauté d'abord réunie par sa pratique commune d'un support de presse particulier. Il n'y a pas de règles communes à ces forums si ce n'est que dans la logique du média, qui se trouve engagée en tant que lieu où se déroule la discussion, un modérateur est nécessaire pour délimiter un cadre et un mode d'expression, pour permettre les échanges et éviter les confrontations trop violentes. Ce type de forums ne s'inscrit pas dans la logique d'une "cour de récréation virtuelle", mais comme un 
espace d'échange de points de vue tel que cela pourrait se dérouler au cours d'une conversation de café ou dans une salle de réunion.

Ici le feed-back n'est pas tant orienté vers le support de presse qu'entre ses usagers. Les forums forment d'abord des espaces nouveaux créant du lien social à travers la rencontre et l'échange de points de vue qui sinon n'auraient pas trouvé à se concrétiser si facilement. Et en tout cas pas avec cette population. Ils permettent la rencontre et la confrontation de personnes éloignées sur un plan géospatial. En revanche, les forums ne jouent guère de rôle dans la mixité sociale et culturelle au sens où ils mettent en contact des individus reliés par des pratiques sociologiques communes. Le brassage est donc relativement limité même si l'on peut considérer qu'il est plus aisé d'entrer en relation par ce biais moins impliquant et qui permet de préserver l'anonymat plutôt que prendre part à une discussion publique, voire de s'exprimer à la table familiale.

Le forum renouvelle et démultiplie les espaces d'intervention. accordés avec parcimonie dans les colonnes des journaux et sur les ondes et les écrans. En effet, par souci de se rapprocher de son public et pour essayer de mieux représenter la diversité des opinions, les médias depuis longtemps se sont ouverts à la parole de l'usager. Cette démarche prolonge les devoirs édictés par la loi concernant le droit de réponse. Il s'agit ici de permettre une prise de parole qui aide à donner du média une image de proximité avec son public. Dans un premier temps la parole était confiée presque exclusivement à des personnalités légitimées par la logique sociale, aux moyens de tribunes et libres propos, puis, la radio, en particulier a initié depuis un quart de siècle des espaces d'intervention où la légitimité provient seulement du fait que l'on soit auditeur du média. La notion de forum démultiplie cette prise de parole publique et surtout transforme la logique de l'intervention : on ne s'exprime plus de façon individuelle vers le reste du public maintenu dans une posture de passivité mais on échange entre usagers.

De la même façon les chat permettent un dialogue en direct entre usagers et avec la rédaction. Il est aussi possible d'instituer des espaces d'échange avec des personnalités. Dans ce cas, le journaliste délègue de façon temporaire sa fonction médiatrice, au profit d'une communauté d'usagers-internautes. Il peut lui rester cependant une responsabilité dans le tri des questions et des interventions.

Les forums contribuent donc à la ré-appropriation par les usagers de leurs médias. 


\section{Notation des articles : le paradoxe de l'invité}

Pour compléter cette analyse, il importe aussi de prendre en compte une forme encore anecdotique des nouvelles pratiques liées à Internet : la notation des articles. Cela concerne principalement les sites de presse en ligne et vient d'une recherche de donner toujours plus satisfaction à l'utilisateur. Celui-ci est amené à donner son avis à travers un système de notation beaucoup plus simple à pratiquer que l'intervention, la contestation, et partant l' analyse critique. Le "lecteur censeur" se contente d'exprimer une opinion ramenée à sa plus simple expression à travers un barème de notes. Dans certains cas, il est demandé de noter chaque article lu, dans d'autres cas, comme le site du Monde, il s'agit de produire une sorte de palmarès des articles les plus appréciés. Cela peut s'afficher comme une volonté de se rapprocher des usagers et comme un moyen de valoriser les sujets les plus importants, mais cette démarche qui crée de fait une nouvelle forme de feed-back avec le média, infantilise ou marchandise la relation à l'information. Nicholas Negroponte proposait déjà ce type de relation grâce à Internet ${ }^{1}$ : pour lui chaque article devrait être payé à la lecture et l'auteur verrait donc sa rémunération évoluer en fonction du degré de satisfaction qu'il est capable de produire. Une façon de valoriser les meilleurs et les plus connus des journalistes. Il fait ainsi fi de l'environnement rédactionnel qui porte une signature : difficile d'atteindre la notoriété par soi-même sans profiter de la légitimité et de la force de frappe d'un support.

Surtout cela impose un rapport encore plus marchandisé avec les usagers et de compétition entre les journalistes : pour avoir de "bonnes notes" ne faut-il pas privilégier certains sujets ou écrire dans une certaine orientation?

De façon assez étonnante ce type de pratique, réalisée aussi par des journalistes produisant seul des lettres d'information et demandant à ce que l'usager juge son propre travail et distingue entre des articles rédigés par une même personne, n'a pas créé de véritables remous. Comme si le primat de l'usager sur le libre arbitre du journaliste était devenu une règle.

I N. NEgroponte, L'Homme numérique, Paris, Eyrolles, 1997. 
Il importe aussi de noter que les usagers se prêtent volontiers à cet exercice dans lequel ils perçoivent l'opportunité de peser sur les choix éditoriaux du support.

Ce mode d'intervention créé aussi une relation nouvelle, une dépendance, entre usagers et médias et prolongent les enquêtes "vulu"' et les baromètres de satisfaction mis en place depuis le début des années 1960 dans les médias, et d'abord dans la presse écrite. L'objectif étant toujours, ce qui tient de la logique de l'affrontement concurrentiel, de chercher à être en phase avec la demande, avec les attentes du public, pour se maintenir dans le marché.

On peut ainsi former l'hypothèse que la mise en ligne des médias a produit de nouveaux comportements des usagers et des journalistes. Cela a favorisé le développement de nouvelles relations, de nouveaux feed-back, plaçant l'usager plus au centre du média.

La "sociologie des usages"2 nous a montré depuis longtemps l'inventivité presque sans limites des utilisateurs d'une technologie naissante. Le corps social met en place une capacité particulièrement fertile à détourner chaque dispositif technique, à prendre des chemins de traverses face aux règles, y compris d'usage, que les premiers concepteurs souhaitaient leur imposer. La société dans sa pluralité peut aussi devenir acteur en inventant des pratiques non prévues par les initiateurs premiers.

Tout processus d'innovation, à plus forte raison dans le domaine du média, de la relation aux autres, génère un processus d'appropriation progressive par le public, qui se pose comme une deuxième naissance. C'est cette deuxième naissance qui le plus souvent fixe l'avenir de l'innovation, son destin, sa pérennité.

En intégrant les sites de presse dans le cursus de leur quotidien, en le reliant à leur culture d'appartenance, une nouvelle relation au média, les usagers de la communication transforment progressivement leur relation à la sphère de l'information. Ces nouveaux utilisateurs, qui ne sont encore qu'une minorité, banalisent la sphère médiatique, mais l'ouvrent aussi sur un rapport d'échange avec les récepteurs, et inventent une nouvelle relance sociale à partir du socle fédérateur du

1 J. Douel, Le journal tel qu'il est lu. Paris, Éditions du CFPJ, 1981.

2 P.A. Mercier et Y. Toussaint, "Les usages", in P. Musso (dir.), Communiquer demain, Datar/Éditions de l'Aube, 1994. 
site fondé sur une image et une crédibilité acquise le plus souvent dans l'univers des médias traditionnels. 